Pacific Journal of Mathematics

COMPACT SEMIGROUPS WITH SQUARE ROOTS 


\title{
COMPACT SEMIGROUPS WITH SQUARE ROOTS
}

\author{
JANE M. DAY
}

\begin{abstract}
Suppose that $S$ is a finite dimensional cancellative commutative clan with $E=\{0,1\}$ and that $H$ is the group of units of $S$. We show that if square roots exist in $S / H$, not necessarily uniquely, then there is a closed positive cone $T$ in $E^{n}$ for some $n$ and a homomorphism $f:(T \cup \infty) \times H \rightarrow S$ which is onto and one-to-one on some neighborhood of the identity. $T \cup \infty$ denotes the one point compactification of $T$.
\end{abstract}

K. Keimel proved in (6), and Brown and Friedberg independently in (1), that if $S / H$ is uniquely divisible, then it is isomorphic to $T \cup \infty$ for some closed positive cone $T$. Brown and Friedberg went on to show that if $S$ is uniquely divisible, then $S$ is isomorphic to the Rees quotient $((T \cup \infty) \times H) /(\infty \times H)$. What we do here is to weaken their hypothesis to assume just square roots in $S / H$ and conclude that $S$ is isomorphic to some quotient of such $(T \cup \infty) \times H$, which will be a Rees quotient if square roots are unique in $(S / H) \backslash 0$, but in general need not be Rees. ${ }^{1} f((T \cup \infty) \times 1)$ is a subclan of $S$ and a local cross section at 1 for the orbits of the group action $H \times S \rightarrow S$ (which equal $\mathscr{C}$ classes here), but an example shows that it need not be a full cross section. Also, square roots exist (uniquely) in $S$ if and only if they exist (uniquely) in $S / H$ and $H$.

The proof consists essentially of showing that the ingenious constructions of (1) can still be done under the weaker hypothesis, in a sufficiently small neighborhood of $H$.

For basic information about semigroups, see (5), (8) or (9). The real intervals $(0,1]$ and $[0,1]$ are semigroups under usual real multiplication; as in (5), a one parameter semigroup is a homomorph of $(0,1]$, and we also define here a closed one parameter semigroup to be a nonconstant homomorph of $[0,1]$.

The Lemmas (I)-(III) are variations on standard themes so we omit proofs. (See (1), (3), (4), B-3 of (5), (6) and (7).) Throughout this paper let $S$ be a clan with exactly two idempotents, a zero and an identity denoted by 0 and 1 respectively.

( I ) If $R$ is a one parameter semigroup in $S$ which is not contained in $H$ and is not equal to 0 , then $R \cup 0$ is a closed one parameter semigroup and an are with endpoints 0 and 1 . Let $\phi:(0,1] \rightarrow R$ be the homomorphism that defines $R$; if $x=\phi(t) \in R$ and $k \geqq 0$, we write

${ }^{1}$ Keimel has concurrently proved a further generalization, by a different method, assuming instead of cancellation that $x \times H \rightarrow x H$ is one-to-one for all $x$ near $H$. 
$x^{k}$ for $\phi\left(t^{k}\right)$, and if $x \neq 0,1$, each $y \in R \backslash 0$ equals $x^{k}$ for unique $k$.

(II) If $H$ is normal and every element of $S / H$ has a square root in $S / H$, then for each $x \in S$ there exists a closed one parameter semigroup in $S$ intersecting $H x$.

(III) Let $T$ be a commutative uniquely divisible clan with group of units $H(T)$ and $E=\{0,1\}$, and let $V$ be a set containing a neighborhood of 1 in $T$ such that $T \backslash V$ is an ideal. If $S$ is commutative and $\psi^{\prime}: V \rightarrow S$ is a continuous function such that $\psi^{\prime}(V \backslash H(T)) \cap H=\square$ and $\psi^{\prime}(x y)=\psi^{\prime}(x) \psi^{\prime}(y)$ whenever $x, y, x y \in V$, then $\psi^{\prime}$ can be extended to a homomorphism $\psi$ on all of $T$ by defining $\psi(0)=0$ and $\psi\left(x^{n}\right)=$ $\psi^{\prime}(x)^{n}$ for each $x \in V$ and positive integer $n$.

The definition of independent family which follows agrees with the algebraic independence used in [1] when $H$ is trivial and $W=S \backslash 0$, and that notion is due to Clark [2]. We include $H$ in our definition so that we do not have to handle the case of $S$ with trivial $H$ separately first, and we define independence in neighborhoods of $H$ rather than in $S$ in order to apply the concept effectively to a clan with nonunique roots.

An independent family in $S$ is a finite family $\left\{R_{1}, \cdots, R_{n}\right\}$ of closed one parameter semigroups in $S$ such that there exists a neighborhood $W$ of $H$ with the property that for every partition of the set $\{1, \cdots, n\}$ into two nonnull disjoint sets $A$ and $B$, this is true:

$$
\underset{i \in A}{P}\left\{R_{i}\right\} \cap\left(\underset{i \in B}{P}\left\{R_{i}\right\}\right) H \cap W \subset H .
$$

We will also describe this situation by saying that $\left\{R_{1}, \cdots, R_{n}\right)$ is independent in $W$. We adopt the convention that if $X=\square$, then $P_{i \in X}\left\{x_{i}\right\}=1$, for $x_{i}$ 's which are elements or subsets of $S$. $S$ will be called cancellative if $x, y, z \in S$ and $x y=x z \neq 0$ implies $y=z$.

We will make frequent use of the following facts. $F(V)$ denotes boundary of $V$. Any neighborhood of $H$ in compact $S$ contains a neighborhood $V$ of $H$ such that $S \backslash V$ is an ideal (A-3.1, (5)), and if $V$ is a set such that $S \backslash V$ is an ideal, then

$$
0 \notin V, V=V H, F(V)=F(V) H,
$$

$S \backslash V^{*}$ is an ideal if nonempty, and $x y \in V$ implies $x, y \in V$. If $J$ is a closed ideal in compact $S$, shrinking $J$ to a point gives a new compact semi-group denoted $S / J$ and called the Rees quotient of $S$ by $J$, and the natural map $S \rightarrow S / J$ is a homomorphism.

Part (i) of the lemma below is analogous to 1.4 of (1); part (ii) shows that the homomorphisms $\phi: S \backslash 0 \rightarrow E^{n}$ and $\beta: S \backslash 0 \rightarrow H$ constructed in (1) can still be constructed here on a sufficiently small neighborhood of $H$. Dim $S$ means inductive dimension of $S$. 
Lemma. Let $S$ be a cancellative commutative clan with $E=\{0,1\}$ and let $W$ be a closed neighborhood of 1 such that $S \backslash W$ is an ideal.

(i) If $\left\{R_{1}, \cdots, R_{n}\right\}$ is an independent family in $W$, and if $x_{1} x_{2} \cdots x_{n} h=x_{1}^{\prime} x_{2^{\prime}} \cdots x_{n}^{\prime} h^{\prime} \in W$, where $x_{i}, x_{i}^{\prime} \in R_{i}$ for each $i$ and $h, h^{\prime} \in H$, then $x_{i}=x_{i}^{\prime}$ for each $i$ and $h=h^{\prime}$; consequently $\operatorname{dim} S \geqq n$.

(ii) Suppose $\operatorname{dim} S \leqq N$ or $\operatorname{dim} S / H \leqq N$ and that $S / H$ has square roots. Then there exists a maximal independent family $\left\{R_{1}, \cdots, R_{n}\right\}$ of closed one parameter semigroups in $S$, and a closed neighborhood $U$ of $H$ may be chosen so that $S \backslash U$ is an ideal and if $x \in U, x$ satisfies this condition.

(ł) There exists a unique partition $(A, B)$ of $\{1, \cdots, n\}$ and unique elements $x_{i} \in R_{i}$ and $h \in H$ such that $i \in B$ whenever $x_{i}=1$ and $x\left(P_{i \in A}\left\{x_{i}\right\}\right)=\left(P_{i \in B}\left\{x_{i}\right\}\right) h \in W$.

Proof. (i) Since $R_{i}$ is a closed one parameter semigroup and $x_{i} \neq 0$, we may factor $x_{i}$ or $x_{i}^{\prime}$ for each $i$ and then commute and cancel in the equality given to get $0 \neq P_{i \in A}\left\{r_{i}\right\}=\left(P_{i \in B}\left\{r_{i}\right\}\right) h^{\prime} h^{-1}$ for some partition $(A, B)$ of $\{1, \cdots, n\}$. These points lie in $W$ so by independence, $r_{i}=1$, hence $x_{i}=x_{i}^{\prime}$, for each $i$, and thus $h=h^{\prime}$ also. There is a closed neighborhood $V$ of 1 such that $V^{n} \subset W$, and then the multiplication function $\left(R_{1} \cap V\right) \times \cdots \times\left(R_{n} \cap V\right) \rightarrow S$ is a homeomorphism so $S$ contains an $n$-cell.

(ii) If $\operatorname{dim} S \leqq N$, then a maximal independent family exists by (i). If $\operatorname{dim} S / H \leqq N$ instead, $S / H$ is cancellative since $S$ is, so (i) can be applied to $S / H$ to get a maximal independent family in $S / H$; a closed one parameter semigroup in $S$ projects to a closed one parameter semigroup in $S / H$ by (I), and it is easy to see that an independent family in $S$ projects to one in $S / H$, so $S$ can have no larger independent family than $S / H$ does.

Now choose a maximal independent family $\left\{R_{1}, \cdots, R_{n}\right\}$ in $S$, and choose $W$ smaller if necessary so that the $R_{i}$ 's are actually independent in a neighborhood of $H$ containing $W^{2}$.

To prove the uniqueness assertion of $(\nmid)$, suppose that

$$
x\left(\underset{i \in A}{P}\left\{x_{i}\right\}\right)=\left(\underset{i \in B}{P}\left\{x_{i}\right\}\right) h \in W \text { and } x\left(\underset{i \in A^{\prime}}{P}\left\{x_{i}^{\prime}\right\}\right)=\left(\underset{i \in B^{\prime}}{P}\left\{x_{i}^{\prime}\right\}\right) h^{\prime} \in W,
$$

as described in $(\nmid)$. Then

$$
\left(\underset{i \in A}{P}\left\{x_{i}\right\}\right)\left(\underset{i \in B^{\prime}}{P}\left\{x_{i}^{\prime}\right\}\right) h^{\prime}=\left(\underset{i \in A^{\prime}}{P}\left\{x_{i}^{\prime}\right\}\right)\left(\underset{i \in B}{P}\left\{x_{i}\right\}\right) h \in W^{2} ;
$$

for each $i$, collect into one term the $x_{k}$ 's with $k=i$, on each side, and suppose there exists $j \in A \cap B^{\prime} ; j \in A$ implies that the factor on the left which is an element of $R_{j}$ is not 1 , and it has to equal one of the factors on the right by (i); therefore $j$ has to be in $A^{\prime}$ or in $B$, because by independence an element of $\left(R_{j} \cap W^{2}\right) \backslash 1$ cannot arise 
from multiples of elements of $R_{i}$ 's for $i \neq j$. But $j \in B$ implies $j \notin A$ and $j \in A^{\prime}$ implies $j \notin B^{\prime}$, both contradictions. So $A \cap B^{\prime}$ must be empty, similarly $A^{\prime} \cap B$ is empty, hence $(A, B)=\left(A^{\prime}, B^{\prime}\right)$. Now apply (i).

Now let $R$ be any closed one parameter semigroup in $S$.

$$
\left\{R, R_{1}, \cdots, R_{n}\right\}
$$

is not independent in any neighborhood of $H$ (where $R$ and $R_{i}$ are each counted if $R=R_{i}$ for some $i$ ), so there is a particular partition $\left(A_{R}, B_{R}\right)$ of $\{1, \cdots, n\}$ such that $T=R P \cap Q H$ contains points arbitrarily near $H$ in $S \backslash H$, where $P=P_{i \in A}\left\{R_{i}\right\}$ and $Q=P_{i \in B_{R}}\left\{R_{i}\right\} . \quad T$ is also a compact semigroup, so it contains a connected subsemigroup from 1 to 0 (B-4.9, (5)). $F(W)$ separates 0 and 1 in $S$, hence we may select $x_{R} \in R$ such that $x_{R} P \cap Q H \cap F(W) \neq \square$. Every $x \geqq x_{R}$ in $R$ satisfies $(\nmid)$ since the complement of an ideal in $R$ is connected and $\{x \in R \mid x P \cap Q H \subset S \backslash W\}$ is an ideal of $R$. It follows that every $x \geqq x_{R}$ in $R H$ satisfies $(\nmid)$ also.

If we can find a closed neighborhood $U$ of $H$ such that $x_{R} \notin U$ for each closed one parameter semigroup $R$ in $S$, then every $y \in U$ lies in some $R H$ by (II), $U$ may be chosen smaller so that $S \backslash U$ is an ideal, and then every $y \in U$ satisfies $(\nmid)$ by the preceding remark. Suppose no such $U$ exists, so there is a net $\left(x_{R}\right)$ of the $x_{R}$ 's clustering at some element of $H$; since there exist only a finite number of partitions of $\{1, \cdots, n\}$, we may suppose that for one particular partition $(A, B)$ and for each $x_{R}$ in the net, $\left(A_{R}, B_{R}\right)=(A, B)$. Then, since $F(W)=F(W) H$, any cluster point of $\left(a_{R}\right)$ is an element of

$$
\underset{i \in A}{P}\left\{R_{i}\right\} \cap\left(\underset{i \in B}{P}\left\{R_{i}\right\}\right) H \cap F(W) ;
$$

but this set is empty (by definition if $A=\square$, and if $A \neq \square$, by independence in $W$ ).

Euclidean $n$-space, denoted $E^{n}$, is a semigroup under vector addition with the origin as identity. If $P^{*}$ is the set of nonnegative real numbers, $N$ the set of negative real numbers, and juxtaposition denotes scalar multiplication, a closed positive cone in $E^{n}$ is defined to be a closed subsemigroup $T$ of $E^{n}$ such that $P^{*} T \subset T$ and $N T \cap$ $T=(0, \cdots, 0)$. The one point compactification $T \cup \infty$ of a nontrivial closed positive cone $T$ is a continuum and becomes a clan with exactly two idempotents, a zero and an identity, when addition is extended by defining $z+\infty=\infty+z=\infty$ for each $z \in T \cup \infty$, and such clans are uniquely divisible (where the " $n$th root" of $z$ would be $(1 / n) z$ since the operation is addition).

THEOREM. Suppose that $S$ is a commutative cancellative clan with $E=\{0,1\}$, such that every element of $S / H$ has a square root in $S / H$. 
If $\operatorname{dim} S \leqq N$ or $\operatorname{dim} S / H \leqq N$, then there is a closed positive cone $T$ in $E^{n}$ and an onto homomorphism $f:(T \cup \infty) \times H \rightarrow S$ which is a homeomorphism of some neighborhood of the identity onto a neighborhood of the identity in $S$. $f$ maps $(T \cup \infty) \times 1$ to a subclan $T^{\prime}$ which is a local cross section at 1 for the natural projection homomorphism $S \rightarrow S / H$.

Proof. Let $W, U$ and $\left\{R_{1}, \cdots, R_{n}\right\}$ be as in (ii) of the Lemma and let $x_{i} \in R_{i} \cap F(U)$ for each $i$. These $x_{i}$ 's will remain fixed throughout the proof, and since $x_{i} \neq 0,1$, by (I) each element of $R_{i} \backslash 0$ equals $x_{i}^{t}$ for a unique nonnegative real number $t$. This together with (ii) of the Lemma implies that for each $x \in U$, there are a unique partition $(A, B)$ of $\{1, \cdots, n\}$, unique real numbers $t_{1}, \cdots, t_{n}$, and unique $h \in H$ such that $x\left(P_{i \in A}\left\{x_{i}^{t}\right\}\right)=\left(P_{i \in B}\left\{x_{i}^{t} i\right\}\right) h \in W$ and $i \in B$ if $t_{i}=0$; following the notation of (1), let $\varepsilon_{i}=1$ if $i \in B$ and $\varepsilon_{i}=-1$ if $i \in A$, let $\phi(x)=\left(\varepsilon_{1} t_{1}, \cdots, \varepsilon_{n} t_{n}\right)$, and let $\beta(x)=h$. Arguments just like those in (1) show that $\phi \times \beta$ is a homeomorphism, if one uses at judicious spots the facts that $W$ is compact and that $S \backslash W$ is an ideal. Since $S$ is commutative, $\phi$ and $\beta$ are homomorphisms as far as they go.

Let $T=P^{*} \phi(U)$. We show next that $\phi(U)$ contains a neighborhood of the origin in $T$ and that $T$ is a closed positive cone in $E^{n}$. First, $T=P^{*} \phi(F(U))$ because each closed one parameter semigroup in $S$ intersects $F(U)$, so $T$ is closed in $E^{n}$ because in general if $A$ is closed in $P^{*}$ and $S$ is compact in $E^{n}$ and does not contain the origin, then $A B$ is closed. For this same reason, [1, $) \dot{\phi}(F(U))$ is closed, hence its complement in $T$ is a neighborhood of the origin in $T$ and also is a subset of $\phi(U)$ because $k \phi(x)=\phi\left(x^{k}\right)$ and $x \in U$ implies $x^{k} \in U$, for $k \in[0,1)$. Since $\phi(U)$ contains a neighborhood of the origin in $T$ and $\phi$ preserves multiplication on $U, T$ is a subsemigroup of $E^{n}$. To see that $N T \cap T$ is the origin it suffices to prove that $(-1) \phi(U) \cap$ $\phi(U)$ is, so suppose $x, x^{\prime} \in U$ and $\phi(x)=(-1) \phi\left(x^{\prime}\right)=\left(t_{1}, \cdots, t_{n}\right)$. Then for some $h, h^{\prime} \in H, x\left(P_{i \in A}\left\{x_{i}^{t_{i}}\right\}\right)=\left(P_{i \in B}\left\{x_{i}^{t_{i}}\right\}\right) h \in W$ and $x^{\prime}\left(P_{i \in B}\left\{x_{i}^{t}\right\}\right)=$ $\left(P_{i \in A}\left\{x_{i}^{t}\right\}\right) h^{\prime} \in W$. Substituting from the first equation into the second and cancelling gives $x^{\prime} x h^{-1}=h^{\prime}$, hence $x, x^{\prime} \in H$, hence $\phi(x)$ is the origin as required.

Now define $\psi: \phi(U) \rightarrow S$ by $\psi(z)=(\phi \times \beta)^{-1}(z, 1)$. $\psi$ is a homeomorphism into and, if $U$ is chosen small enough that $\phi$ is actually defined on $U^{2}, \psi$ preserves multiplication on $\phi(U)$ also. $T$ is uniquely divisible so by (III), $\psi$ may be extended to a homomorphism of $T$ into $S$. Now define $f:(T \cup \infty) \times H \rightarrow S$ by $f(z, h)=\psi(z) h . \quad f$ is a homomorphism because $\psi$ is and $S$ is commutative, and it is a homeomorphism of $\dot{\phi}(U) \times H$ onto $U$ because there it equals $(\phi \times \beta)^{-1}$. (We cannot use (III) to define $f$ directly as an extension of $(\dot{\phi} \times \beta)^{-1}$, because $H$ need not be uniquely divisible.) Since the image of $f$ is a 
subclan of $S$ which contains a neighborhood of $H$ and since $S$ is divisible, $f$ is onto. Therefore $T^{\prime} H=S$ so $T^{\prime \prime} \rightarrow S / H$ is onto and the rest is clear.

In a semigroup with zero, a nilpotent is a nonzero element some finite power of which is zero.

COROLlary. Let everything be as in the theorem.

(i) If square roots are unique in $(S / H) \backslash 0$ (but there could be nilpotents) then $f$ is one-to-one on the complement of $f^{-1}(0)$, hence $f$ induces an isomorphism from the Rees quotient $((T \cup \infty) \times H) / f^{-1}(0)$ onto $S$ and also $T^{\prime}$ is a full cross section for $H \times S \rightarrow S$. If square roots are unique in all of $S / H$ (so there are no nilpotents) then $f^{-1}(0)=\infty \times H$, so $S$ is isomorphic to $((T \cup \infty) \times H) /(\infty \times H)$ (Theorem 2.2 of (1)).

(ii) Square roots exist (uniquely) in $S$ if and only if they exist (uniquely) in $H$ and $S / H$.

Proof. Let $p: S \rightarrow S / H$ be the natural map. If $f(t, h)=f(s, g) \neq 0$, then $f(t, 1) h=f(s, 1) g$ hence $p f(t, 1)=p f(s, 1)$. Uniqueness of roots in $(S / H) \backslash 0$ implies $p f(k t, 1)=p f(k s, 1)$ for all $k \geqq 1$ at least, and $p f$ is one-to-one near the identity by the theorem, hence $k t=k s$ must be true for $k$ sufficiently small. Therefore $t=s$ and cancelling $f(t, 1)$ now gives $h=g$ also. The rest is clear.

Example 1. This was also discovered by D. Brown and M. Friedberg (and communicated orally to this author). It is a cancellative commutative clan $S$ with $E=\{0,1\}$ and trivial group of units, which has no nilpotents and is divisible but not uniquely divisible; in fact, any two distinct one parameter semigroups in $S$ are independent near 1 and have no nondegenerate arc in common, but can intersect infinitely. Thus $S$ is not a Rees quotient of any compactified cone. The author is indebted to Kermit Sigmon for the elegance of this description of the example.

Let $T$ be the closed first quadrant of $E^{2}$, let $D$ be the closed unit disc in the complex plane with usual complex multiplication, and define $g: T \cup \infty \rightarrow D$ by $g(x, y)=e^{-(x+y)+(x-y) \pi i}$ and $g(\infty)=0 . \quad g$ is a homomorphism by (III), so $S=g(T \cup \infty)$ is a clan, it has $E=\{0,1\}$, is topologically a 2-cell, and is an egg-shaped subset of $D$ with large end at 1 and small end at $-1 / e . \quad S$ is commutative, cancellative and free of nilpotents since $D$ is, has roots of all orders since $T U \propto$ does, and square roots are not unique since $\phi(1,0)=\phi(0,1)$ but $\phi(1 / 2,0) \neq$ $\phi(0,1 / 2)$.

$S$ can also be visualized without the aid of $D$ : there is a congruence $\sim$ on $T \cup \infty$ such that $S$ is isomorphic to $(T \cup \infty) / \sim$ : it is 
the smallest congruence which identifies $(0,1)$ and $(1,0)$, and dividing by it has the effect geometrically of rolling up $T \cup \infty$ into a cone with pointed end at $\infty$.

EXAMPLE 2. This will show that the subclan $T^{\prime \prime}$ of the theorem need not be a full cross section for $H$ orbits, i.e., $\mathscr{H}$ classes. Let $T \cup \infty$ be as in the previous example, let $G$ be the circle group with usual complex number notation, and let $Q$ be the product semigroup $(T \cup \infty) \times G$. We will twist the $\mathscr{H}$ class of $(0,1,1)$ and then identity it with the $\mathscr{H}$ class of $(1,0,1)$. Formally, let $\sim$ be the smallest closed congruence on $Q$ which identifies $(0,1,1)$ and $(1,0,-1)$, let $S=Q / \sim$, and let $f: Q \rightarrow S$ be the natural projection. Thus if $\Delta$ is the diagonal of $Q \times Q, p=[(0,1,1),(1,0,-1)]$, and $q=[(1,0,-1)$, $(0,1,1)]$, then $\sim$ is the smallest closed symmetric subsemigroup of $Q \times Q$ containing $p \cup \Delta$, and $p q \in \Delta$ so this equals $\Delta(\Gamma(p) \cup \Gamma(q) \cup \Delta)$. Clearly $[(0,1,1),(1,0,1)]$ is not in the semigroup generated by $p \cup$ $q \cup \Delta$, and $\Gamma(p)$ and $\Gamma(q)$ have only one limit point, $\infty$, so this point is not in $\sim$, i.e., $f(0,1,1) \neq f(1,0,1)$. On the other hand, the $\mathscr{H}$ classes in $S$ of these points are equal, because $H=f(0 \times 0 \times G)$ is the group of units of $S$ and $f(0,1,1)=f(1,0,1) f(0,0,-1)$.

$f$ is a homeomorphism on $[0,1) \times[0,1) \times G$, which is a neighborhood of the identity, and we will show below that $S$ is cancellative, so this is exactly the situation of the theorem. However, if $T^{\prime}$ denotes $f((T \cup \infty) \times 1), T^{\prime} \rightarrow S / H$ is not one-to-one.

Interestingly, there actually is a full cross section semigroup for the $H$ orbits of this clan $S$; the problem in the above lies in the definition of $f$-that is, in the choice of the independent closed one parameter semigroups in $S$ :

$$
R_{1}=f([0, \infty] \times 0 \times 1) \text { and } R_{2}=f(0 \times[0, \infty] \times 1)
$$

are independent but do not themselves intersect in some of the $H$ orbits which they both go through. Rechoosing $f$ so that $R_{2}$ actually does intersect $R_{1}$ at the levels where $Q \rightarrow S$ collapses two $H$ orbits to one yields a subclan $T^{\prime \prime}$ of $S$ which is isomorphic to $S / H$. In detail, define $g: Q \rightarrow Q$ by $g\left(x, y, e^{i \theta}\right)=\left(x, y, e^{i(\theta+\pi y)}\right)$, let $f^{\prime}=f g$, and let $T^{\prime \prime}=$ $f^{\prime}((T \cup \infty) \times 1)$. To see that $T^{\prime \prime} \rightarrow S / H$ is one-to-one, suppose

$$
f g(x, y, 1)=f g\left(x^{\prime}, y^{\prime}, 1\right) f g\left(0,0, e^{i \theta}\right) \neq 0 .
$$

We will prove $e^{i \theta}=1$. In $g(x, y, 1)=g\left(x^{\prime}, y^{\prime}, e^{i \theta}\right)$ then we are done because $g$ is one-to-one, so suppose $g(x, y, 1) \neq g\left(x^{\prime}, y^{\prime}, e^{i \theta}\right)$. $f$ identifies these points and not to 0 so for some $n,\left(\left(g(x, y, 1), g\left(x^{\prime}, y^{\prime}, e^{i \theta}\right)\right) \in \Delta p^{n}\right.$. An arbitrary point of $\Delta p^{n}$ is of the form $\left(\left(s, n+t, e^{i \phi}\right),\left(n+s, t, e^{i(\dot{\phi}+n \pi)}\right)\right)$ for some $s, t$ and $\phi$, so we conclude $x^{\prime}=x+n, y=y^{\prime}+n, e^{i \approx y}=e^{i \dot{\phi}}$, 
and $e^{i\left(\theta+\pi y^{\prime}\right)}=e^{i(\phi+n \pi)}$. These imply $e^{i\left(\theta+\pi y^{\prime}\right)}=e^{i \pi y^{\prime}}$, so $e^{i \theta}=1$ as asserted. From this it follows at once that $T^{\prime \prime} \rightarrow S / H$ is one-to-one and in fact that $S$ is isomorphic to $\left(T^{\prime \prime} \times H\right) /(\infty \times H)$.

Now it is easy to show $S$ cancellative, for it suffices to prove that $T^{\prime \prime}$ is, so suppose $f g(x, y, 1) f g(s, t, 1)=f g\left(x^{\prime}, y^{\prime}, 1\right) f g(s, t, 1)$. It follows that $x+s+n=x^{\prime}+s$ and $y+t=y^{\prime}+t+n$ for some $n$, hence $x+n=x^{\prime}$ and $y=y^{\prime}+n . \quad f g(x, y, 1)=f g\left(x^{\prime}, y^{\prime}, 1\right)$ now is clear.

It seems at least possible that the technique used here for rechoosing $f$ might work in general, so that there is always a full cross section semigroup for $S \rightarrow S / H$ when $S$ is a homomorph of the direct product of $H$ and a closed positive cone.

It also seems reasonable to conjecture that the theorem is still true with only $H$ normal and $S / H$ commutative, instead of $S$ commutative. Under these weaker conditions $\phi$ and $\beta$ still exist, but $\beta$ need not be a homomorphism unless the $R_{i}$ 's commute with one another and with $H$; using Theorem VI of (5), it is possible to choose a maximal independent set in the centralizer of $H$, but the problem of choosing the $R_{j}$ 's to commute with one another also remains unsolved.

\section{REFERENCES}

1. D. R. Brown and M. Friedberg, Representation theorems for uniquely divisible semigroups, Duke Math. J., 35 (1968), 341-352.

2. C. E. Clark, Homomorphisms of compact semigroups, Dissertation, Louisiana State University, 1966.

3. A. H. Clifford, Connected ordered topological semigroups with idempotent endpoints, I, T. A.M.S., 91 (1958), 80-98.

4. W. M. Faucett, Compact semigroups irreducibly connected between two idempotents, P.A.M.S., 6 (1955), 741-747.

5. K. H. Hofmann and P. S. Mostert, Elements of Compact Semigroups, C. E. Merrill, Columbus, 1966.

6. K. Keimel, Eine Exponentialfunktion für kompakte abelsche Halbgruppen, Math. Zeit., 96 (1967), 7-25.

7. P. S. Mostert and A. L. Shields, On the structure of semigroups on a compact manifold with boundary, Ann. Math., 65 (1957), 117-143.

8. A. B. Paalman-de Miranda, Topological semigroups, Mathematisch Centrum, Amsterdam, 1964.

9. A. D. Wallace, Project mob, Department of Mathematics, University of Florida, 1965.

Received August 2, 1969, and in revised form June 23, 1970. The author is grateful to the American Association of University Woman for a postdoctoral fellowship which supported part of this research.

College of Notre Dame 


\title{
PACIFIC JOURNAL OF MATHEMATICS
}

\section{EDITORS}

H. SAMELSON

Stanford University

Stanford, California 94305

C. R. HobвY

University of Washington

Seattle, Washington 98105

\section{J. DugunduI}

Department of Mathematics

University of Southern California

Los Angeles, California 90007

RICHARD ARENS

University of California

Los Angeles, California 90024

\section{ASSOCIATE EDITORS}
E. F. BECKENBACH
B. H. Neumann
F. WOLE
K. Yoshida

\section{SUPPORTING INSTITUTIONS}

\author{
UNIVERSITY OF BRITISH COLUMBIA \\ CALIFORNIA INSTITUTE OF TECHNOLOGY \\ UNIVERSITY OF CALIFORNIA \\ MONTANA STATE UNIVERSITY \\ UNIVERSITY OF NEVADA \\ NEW MEXICO STATE UNIVERSITY \\ OREGON STATE UNIVERSITY \\ UNIVERSITY OF OREGON \\ OSAKA UNIVERSITY \\ UNIVERSITY OF SOUTHERN CALIFORNIA
}

\author{
STANFORD UNIVERSITY \\ UNIVERSITY OF TOKYO \\ UNIVERSITY OF UTAH \\ WASHINGTON STATE UNIVERSITY \\ UNIVERSITY OF WASHINGTON

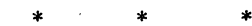 \\ AMERICAN MATHEMATICAL SOCIETY \\ CHEVRON RESEARCH CORPORATION \\ NAVAL WEAPONS CENTER
}

The Supporting Institutions listed above contribute to the cost of publication of this Journal, but they are not owners or publishers and have no responsibility for its content or policies.

Mathematical papers intended for publication in the Pacific Journal of Mathematics should be in typed form or offset-reproduced, (not dittoed), double spaced with large margins. Underline Greek letters in red, German in green, and script in blue. The first paragraph or two must be capable of being used separately as a synopsis of the entire paper. The editorial "we" must not be used in the synopsis, and items of the bibliography should not be cited there unless absolutely necessary, in which case they must be identified by author and Journal, rather than by item number. Manuscripts, in duplicate if possible, may be sent to any one of the four editors. Please classify according to the scheme of Math. Rev. Index to Vol. 39. All other communications to the editors should be addressed to the managing editor, Richard Arens, University of California, Los Angeles, California, 90024.

50 reprints are provided free for each article; additional copies may be obtained at cost in multiples of 50 .

The Pacific Journal of Mathematics is published monthly. Effective with Volume 16 the price per volume (3 numbers) is $\$ 8.00$; single issues, $\$ 3.00$. Special price for current issues to individual faculty members of supporting institutions and to individual members of the American Mathematical Society: $\$ 4.00$ per volume; single issues $\$ 1.50$. Back numbers are available.

Subscriptions, orders for back numbers, and changes of address should be sent to Pacific Journal of Mathematics, 103 Highland Boulevard, Berkeley, California, 94708.

PUBLISHED BY PACIFIC JOURNAL OF MATHEMATICS, A NON-PROFIT CORPORATION

Printed at Kokusai Bunken Insatsusha (International Academic Printing Co., Ltd.), 7-17, Fujimi 2-chome, Chiyoda-ku, Tokyo, Japan. 


\section{Pacific Journal of Mathematics}

\section{Vol. 36, No. $3 \quad$ BadMonth, 1971}

E. M. Alfsen and B. Hirsberg, On dominated extensions in linear subspaces of

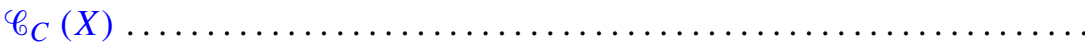

Joby Milo Anthony, Topologies for quotient fields of commutative integral

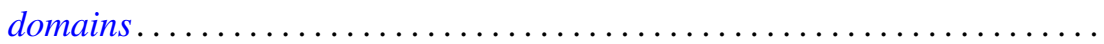

V. Balakrishnan, G. Sankaranarayanan and C. Suyambulingom, Ordered cycle lengths in a random permutation .......................... 603

Victor Allen Belfi, Nontangential homotopy equivalences............... 615

Jane Maxwell Day, Compact semigroups with square roots .............. 623

Norman Henry Eggert, Jr., Quasi regular groups of finite commutative nilpotent algebras .......................................... 631

Paul Erdős and Ernst Gabor Straus, Some number theoretic results ......... 635

George Rudolph Gordh, Jr., Monotone decompositions of irreducible Hausdorff continua .............................................

Darald Joe Hartfiel, The matrix equation $A X B=X \ldots \ldots \ldots \ldots \ldots \ldots . \ldots 69$

James Howard Hedlund, Expansive automorphisms of Banach spaces. II . . . . 671

I. Martin (Irving) Isaacs, The p-parts of character degrees in p-solvable

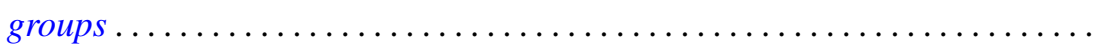

Donald Glen Johnson, Rings of quotients of $\Phi$-algebras ............... 693

Norman Lloyd Johnson, Transition planes constructed from semifield planes....

Anne Bramble Searle Koehler, Quasi-projective and quasi-injective modules.

James J. Kuzmanovich, Completions of Dedekind prime rings as second endomorphism rings...

B. T. Y. Kwee, On generalized translated quasi-Cesàro summability ...

Yves A. Lequain, Differential simplicity and complete integral closure

741

Mordechai Lewin, On nonnegative matrices.

753

Kevin Mor McCrimmon, Speciality of quadratic Jordan algebras ...

Hussain Sayid Nur, Singular perturbations of differential equations in abstract spaces .....................................

D. K. Oates, A non-compact Krein-Milman theorem .

Lavon Barry Page, Operators that commute with a unilateral shift on an invariant subspace...

Helga Schirmer, Properties of fixed point sets on dendrites.

Saharon Shelah, On the number of non-almost isomorphic models of $T$ in a

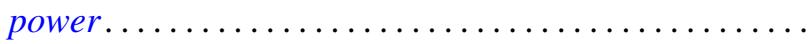

Robert Moffatt Stephenson Jr., Minimal first countable Hausdorff spaces....

Masamichi Takesaki, The quotient algebra of a finite von Neumann algebra 